\title{
A FORUM ON CRUCIAL ISSUES IN THE SIMULATION OF MANUFACTURING SYSTEMS
}

\author{
CHAIR
}

\author{
Averill M. Law \\ Averill M. Law \& Associates \\ P.O. Box 40996 \\ Tucson, Arizona 85717
}

\section{PANELISTS}

\author{
John S. Carson \\ Carson/Banks \& Associates, Inc. \\ 4665 Lower Roswell Road \\ Marietta, Georgia 30068 \\ Kenneth J. Musselman \\ Pritsker Corporation \\ 1305 Cumberland Drive \\ West Lafayette, Indiana 47906
}

\author{
Jerry G. Fox/Stephen K. Halladin \\ Boeing Commercial Airplane Group \\ P.O. Box 3707 \\ Seattle, Washington 98124 \\ Onur M. Ulgen \\ Production Modeling Corporation \\ One Parkland Blvd., Suite 1604 West \\ Dearborn, Michigan 48126
}

Reprinted in part from Industrial Engineering magazine, May 1993, Copyright 1993, Institute of Industrial Engineers, 25 Technology Park/Atlanta, Norcross, Georgia 30092.

\footnotetext{
ABSTRACT

In this panel discussion five leading simulation practitioners discuss a number of critical issues facing a person who is trying to use simulation to design a new system or to improve the performance of an existing system. John Carson, Kenneth Musselman, and Onur Ulgen are consultants, while Jerry Fox/Stephen Halladin are major users of simulation at a company that very extensively employs this technology.

The questions discussed in this article were chosen by the discussants and myself based on our collective years of simulation modeling experience as being both important and of general interest. One of the questions-how to determine the level of model detail--is, perhaps, the single most difficult methodological issue confronting an analyst trying to model a large, complex system. If there is not enough detail in the model, then it will probably be invalid and produce erroneous results. On
}

the other hand, an overly detailed model may result in missed deadlines and in excessive computer execution time or memory requirements. Two additional questions address the ingredients for overall project success and how to collect good information/data on the system of interest. Finally, the discussants give their views on where the field of simulation modeling is headed in the next three years.

This discussion is based in part on a similar paper by Law (1993).

\section{QUESTIONS FOR DISCUSSION}

What are the most important ingredients for a successful simulation project?

\section{John Carson:}

The first key is effective communication between the model developer and the client's staff, including those working on behalf of the client. Among the client's staff who may have knowledge of some aspect of the system being simulated include engineers, supervisors, workers, maintenance people, and management. For new systems and retrofits of existing systems, those working on the 
client's behalf may include systems integrators, controls engineers, and equipment manufacturers.

The second key is management support and commitment. Simulation may require a major effort and should be undertaken only if the potential benefits justify the effort, and management is willing to commit the necessary resources.

The third key is the skills of the team put together to do the project. These include the model developer and a client liaison, whose job it is to ferret out the person with the answers to the modeler's questions. The model developer's skill in asking the right questions and gaining the client's agreement to reasonable simplifying assumptions is one important ingredient.

\section{Jerry Fox/Stephen Halladin:}

We would define a successful simulation project as one in which there is a good working relationship between the simulation analyst and the customer, where simulation is the proper analysis tool, where the process is accurately modeled and analyzed, and results are timely and effectively used by the customer to improve the design or operation of his process.

A good teaming relationship requires commitment on the part of the analyst and customer. The customer must be willing to provide the time and resources to understand the simulation process, carefully outline the objectives of the simulation, provide data and detailed process knowledge for modeling, actively participate in validation of the model, and be committed to effectively using model results, not just justifying his own agenda.

The simulation analyst must have a good understanding of simulation methodology and a mastery of the simulation language to be used. He should have the ability to team with the customer and lead the simulation effort so the objectives of the project are met.

\section{Kenneth Musselman:}

A simulation project is a complex process of interpretive, developmental, and analytical steps. It requires managerial skills to properly set objectives, control expectations, and maintain momentum. It requires technical skills to correctly characterize the input and interpret the output. It also requires modeling skills to effectively capture the essence of the system. Each step of the process is important. Failure to accomplish any one can undermine the project.

Yet, when one examines what makes it all possible, it comes down to one thing--people. Success doesn't come from a new software program or a more discerning statistical test. It doesn't come from a clever modeling trick or a slick presentation. It comes from sound relationships, those that exist between the end user and the modeler, between the consultant and the client, and between the engineer and the manager. If these relationships are truly supportive, then the other ingredients necessary for success will blend nicely. If not, the project will likely loose its cohesion and not achieve its objectives.

\section{Onur Ulgen:}

There are many factors that impact upon the success of a simulation project, including:

(1) Communicating with the client throughout the lifecycle of the simulation project. The client will then know what to expect from the simulation study and be familiar with the assumptions made in the study. This will result in the simulation findings' being used properly by the client.

(2) Involving the client in at least the model validation process, if the client is not available throughout the simulation project.

(3) Completing the project within its time limit, which sometimes requires limiting the level of model detail and making simplifying assumptions. If simulation results play an important role in the decisions to be made, the value of these results decreases rapidly if the results are available after their due date.

(4) Familiarizing the modeler with the system to be modeled, so that proper assumptions are made about the system.

(5) Picking the proper simulation and statistical analysis tools for the problem at hand as well as the modeler involved with the study.

When modeling a large, complex system, how do you decide on a level of model detail that assures both validity and project efficiency (in terms of time, cost, and computer resources)?

\section{John Carson:}

The chief guidelines are provided by the questions to be addressed by the model and a sense of the importance of various variables and subsystems. The model must have sufficient detail to capture the causal connection between the input parameters that will be manipulated and the system performance measures.

At project initiation, the modeler should take the 
client's questions and translate them into specific experiments that can be run on the model. This exercise will help to identify the key input parameters and the key performance measures, and further to identify those subsystems that need detailed modeling.

Other considerations that may influence level of detail include data availability, degree of certainty or uncertainty regarding a subsystem's performance, and of course, expected project time and cost. For example, sometimes a subsystem may be modeled by its output rate (a low level of detail) versus its detailed mechanisms (a higher level of detail).

A model may be valid with many different levels of detail. That is, depending on the level of detail, it may or may not be valid with respect to a given set of questions. Different questions may require more or less detail for particular subsystems.

\section{Jerry Fox/Stephen Halladin:}

The first step in determining the level of model detail is to start with clear concise project objectives. One approach, especially if objectives are soft is to develop a high-level model. The high-level model may have vague assumptions and imprecise data. Sensitivity analysis on high-level models can then identify areas requiring more detail.

As the model is refined, intermediate results are presented to the customer. The importance, relevance, and impact (time and performance) of additional model complexity is then understood by the customer. This continuous communication with the customer ensures that both understand the many assumptions and decisions made and their impacts. We rely on the modelers experience, project objectives, expert opinions, validation requirements, and time limits to define the level of detail. Ultimately the customer and modeler must agree on a level of detail which satisfies the project objectives without sacrificing the accuracy of the answer.

Usually the level of detail between areas, either physical or logical, differs. Areas with greater sensitivity to the objectives of the project require more detail. Modelers should be cautious of enthusiastic shop experts with reams of "useful" data. Modelers often find themselves unnecessarily modeling an area in detail when data is readily available.

Although deciding the level of detail of large complex models is difficult, close communication with the customer, incremental model development, and a focus on project objectives will help ensure a timely, efficient modeling effort. $\underline{\text { Kenneth Musselman: }}$

Every project needs a compass. Without it, a project can drift aimlessly. The compass serves as a frame of reference. This is important when working through ambiguous issues, such as determining an appropriate level of detail.

The best compass is a well-defined set of objectives. This is because the objectives orient the project by providing criteria against which modeling decisions (including level of detail) can be judged.

With the criteria established, the issue of detail can be addressed by working backwards in an orderly manner. First, generate a list of specific questions that support the project's objectives. These questions provide the direction needed to identify the areas in the system of primary importance. Next, determine what output measures are needed to answer these questions. Then, by focusing on these measures and only these measures, the model's scope can be more narrowly defined and its detail reduced.

This leaner model offers two important benefits in practice. First, the modeler can get preliminary results out more quickly, providing for early validation. Second, the smaller model means less program baggage and more modeling freedom. Thus, changes in project direction can be more readily accommodated, saving both time and cost.

\section{Onur Ulgen:}

A model of a large, complex system need not necessarily be complicated. The objectives of the study and the questions to be answered by the model are the main drivers of the level of model detail, assuming needed project resources are available. Note that a complex model of a system is not necessarily a more valid model than a simple model. With a complex model, there are more model data to validate the components of the model as well as the whole model. However, the overall model is not guaranteed to predict system behavior better than a simpler model of the same system, which has been validated using only global system variables.

The basic assumption made here is that there are enough micro- and macro-level data available from the real system so that data-comparative validation techniques are equally applicable for complex and simple models of the system. On the other hand, if only microlevel data are available, a detailed-level model will be desirable. Similarly, if only macro data are available, an aggregate-level model is appropriate. If both types of data are available, the model should be at the simplest level that satisfies the objectives of the study. 
When it comes to project efficiency (assuming appropriate data are available for both simple and detailed models), lack of resources may force the modeler to build a macro-level model even though certain questions may be better answered with a microlevel model. On the other hand, if ample resources are available, model level should depend solely on the questions to be answered by the study. Note that the availability of ample resources should not drive the analyst to build a detailed model if study questions can be answered with a macro-level model.

\section{How do you obtain accurate information and data on} the system being simulated?

\section{John Carson:}

Obtaining accurate information depends on effective communication with the client's staff and others, as previously discussed.

Obtaining accurate data is more problematic and sometimes is not possible beyond subjective estimates. There are the commonly cited sources: computer databases, automatic data collection devices, maintenance records, production records, time studies and sampling studies, interviews (to obtain rough subjective estimates), and equipment specifications (to obtain, for example, machine speeds and expected time to failure).

To obtain accurate data, when you have data at all, you must avoid the dangers, such as modeling all variation as stochastic variation, when in fact the variation may be a function of another variable (e.g., product type); and using a mean value instead of a random variable or a look-up. Keep in mind that bad data often arises from mundane causes, such as typos, gross rounding or aggregation, carelessness during collection, and misunderstanding of the precise data needed.

To avoid using wrong data, I usually recommend a graphical, non-statistical presentation of any data set, to show the client aspects of the data that may not be apparent from a list of numbers. For example, are there outliers, either large or small?

Finally, a sensitivity analysis can identify those variables that are most important and thus need to be estimated most precisely. Roughly estimate the others. Unfortunately, for different scenarios, other variables may become critical, so that you need a sensitivity analysis for every key scenario.

\section{Jerry Fox/Stephen Halladin:}

Impossible? Difficult? Time consuming? These are a few thoughts that come to mind. Sources of information can be overwhelming or non-existent. Data sources include interviews with experts, time studies, manual data records, computer logs, personal observations, documented procedures, vendors, etc. An analyst should not accept information at face value; he must question and understand it. Common sense or sanity checks must be used to determine the value of the information. Spreadsheets and queueing theory models have been used effectively to this end.

An approach taken at Boeing for data collection is to form a small team of experts who support the manufacturing facility. However, larger projects may also require representatives from Manufacturing, Planning, Production Control, Facilities, Industrial Engineering, and Material. Each individual on the team must be committed to understanding simulation, being involved in the data collection, and understanding how the information will be used. When information is collected by others for the analyst, it must be clearly defined so there is no question as to the relevance and accuracy of the data. As the analyst expects the team to understand simulation, the team expects the analyst to understand the process. Personal observation or understanding of the operation is critical. If an analyst relies on area experts without having first-hand knowledge, questions affecting model design may not be asked. For example, a shop might have a two-shift operation, yet certain equipment such as ovens might operate on third shift.

Information accuracy and model credibility are enhanced through a better understanding of the processes and data sources, and frequent communication with the team to validate data and assumptions.

\section{Kenneth Musselman:}

Data collection is best left to those who are most familiar with the process and its data. This, however, does not obviate the project team from responsibility. On the contrary, at a minimum the team should ensure that those responsible for collecting the information understand what is being requested, why it is being requested, and how it is intended to be used. This puts those who are actually collecting the information in a much better position to interpret what needs to be gathered and how it should be filtered.

Keep in mind, models drive what data to collect, but data can drive how models should be built. In practice, it is often more prudent to use available data than to require the collection of new data. Loading the model with what data you do have is often sufficient to test if additional time and energy need to be expended to get better data.

Also, don't be held captive by data you don't have. 
Be willing to make assumptions to maintain progress on the project. Making an assumption is only a problem if you are unwilling to revise it later. Learn to use the model to judge its sensitivity to the assumptions. Then, if found to be sensitive, justification exists to collect more data.

\section{Onur Ulgen:}

If the amount of available system data is inadequate for the purposes of the study, the modeler should directly participate in collecting data whenever possible. If data are to be collected by others, the modeler should at least supervise data-collection activities. If possible, appropriately detailed data should be collected around the clock for a reasonable amount of time. One should keep the base and alternative model data requirements in mind. Note that input as well as output data for the system should be collected for the same time frame, so that it can be used for validation. One should make sure that cause and effect relationships are also identified during the data-collection process. Process flow information and collected data should be discussed with the plant floor engineers, supervisors, and machine operators for further clarification and validation. The functional specification for the project should contain all of this information in a report format.

If the available data on the system appears adequate for the study, the modeler should discuss the conditions under which the data were collected with those responsible. The idiosyncracies in the data should be discussed and outliers, if any, should be identified with possible causes.

In both cases, one should critically review data available or to be collected by an automated datacollection system, and also identify the cause and effect relationships of events in the system. Production reports should be used to cross-check the validity of data collected from automated systems. Similarly, process flow and logic information collected by others should be validated by actual observations and by discussion with plant personnel.

\section{Where do you think the field of simulation modeling is headed in the next three years?}

\section{John Carson:}

There is continual market pressure toward greater ease of use and accessibility for simulation software. To meet this demand, we have seen a new category of software, the simulator, based on a menu-driven user interface and a purported "no programming" point-andclick approach. Unfortunately, what makes these products easy to use, namely their built-in assumptions and pre-programmed constructs, also limits their domain of applicability and the degree of attainable accuracy for a model of a particular system.

The expert user may still prefer the power and flexibility of the full-fledged language, which, however, requires considerable training, expertise, and experience to attain modeling proficiency.

To meet this market demand, and to overcome the dichotomy between the easy-to-use yet limited simulator, and the powerful and flexible yet difficult-to-use language, we have begun to see, and will continue to see over the next few years, simulation software that is extendible and customizable. By extendible, I mean that by using a built-in language, an expert simulationist will be able to build (i.e., program) "macros" or "super blocks" or "templates" that accurately extend and customize the software to particular application domains. From this point, the occasional modeler can build accurate models of his or her system without making over-simplifying assumptions or accepting gross approximations and without programming.

In a similar fashion, supporting activities such as statistical analysis and design of experiments, and data management, are becoming integrated with simulation software, making all aspects of modeling easier and more accessible. This trend will continue.

\section{Jerry Fox/Stephen Halladin:}

We have come to realize through training and consulting work within Boeing that simulation modeling really requires a great deal of expertise. Gains in ease of use through improved software interfaces and built-in modeling constructs have been offset by additional features such as graphics, animation, design of experiments, output analysis, report generators, etc. The wealth of features can overwhelm a new user. The steep learning curve for simulation languages has been offset by simulators and domain-specific applications, which achieve simulation benefits and reduce the expertise required.

In the next three years, we can expect a broader use and application of simulation as more engineering and business schools teach simulation. Growth is expected in enterprise modeling, financial modeling, scheduling, and paper and information flow. Simulation tools will become more visual and intuitive. A more natural way of developing applications, in terms of pictures and objects, which parallels the way we think will be available.

Integration of simulation technology into other computing tools is also occurring. Simulation is being integrated into process modeling tools, CASE, shop-floor 
scheduling, expert systems, and manufacturing cell control software. As the hardware and software costs continue to drop, the market for simulation tools will expand. All aspects of businesses today are realizing that simulation provides a cheap insurance policy, which allows decision makers to make the right decision the first time. The number and variety of tools has grown rapidly over the last six years and this trend should continue.

\section{Kenneth Musselman:}

Most of the activity in simulation over the next few years will fall into three areas: system improvements, simulators, and a common decision domain. System improvements will continue to garner the most attention. Graphical user interfaces, linkages to other, wellestablished software packages, and object-oriented modeling will head the field. Hybrid techniques (e.g., coupling simulation with optimization or artificial intelligence) will be of interest, but will command less attention.

Application-specific simulators will grow in number. Currently, there are few, commercially viable simulators. They address such areas as manufacturing, packaging, material handling, and communications. Yet, the possible application areas are endless. Simulator developments will explode as more interest is shown in new niche markets.

Lastly, manufacturing will put more emphasis on a common decision domain. Regardless of whether the issue is in design, planning, or scheduling, the inference will be based on a common data set that is indigenous to the manufacturing process. The opportunity for realizing performance improvement through such an approach is enormous, as some manufacturers have already discovered. As more recognize this, the demand for continued progress in this area will increase significantly.

\section{Onur Ulgen:}

In the next three years, we are likely to see the following developments:

(1) Simulation software vendors will supply seamless model building environments, which will include both simulation language and simulator features. Simulation software will also include input and output data analyzers, or access to third-party software. There may also be support for the automated development of simulation-based schedulers, including hooks for real-time data acquisition.
(2) Almost all the simulation environments will be based on the object-oriented paradigm.

(3) Seamless robot cell and discrete-event simulation environments will become more popular, which will in turn make multi-level (micro/macro) and hierarchical studies more common.

(4) Life-cycles of simulation models will expand and follow closely the life cycles of actual systems, from conceptual birth to their physical death.

\section{REFERENCE}

Law, A. M. 1993. A forum on crucial issues in simulation modeling, Industrial Engineering 25:32-36.

\section{AUTHOR BIOGRAPHIES}

AVERILL M. LAW is President of Averill M. Law \& Associates (Tucson, Arizona), a company specializing in simulation model building, training, and software. He has been a simulation consultant to more than 80 organizations, including General Motors, IBM, AT\&T, General Electric, Nabisco, Xerox, NASA, the Air Force, the Army, and the Navy. He has presented more than 210 simulation short courses in 12 countries, and delivered more than 100 talks on simulation modeling at technical conferences.

$\mathrm{He}$ is the author (or coauthor) of three books and more than 35 papers on simulation, manufacturing, communications, operations research, and statistics, including the textbook Simulation Modeling and Analysis that is used by more than 34,000 people worldwide. His series of papers on the simulation of manufacturing systems won the 1988 Institute of Industrial Engineers' best publication award. $\mathrm{He}$ is the codeveloper of the UniFit II software package for selecting simulation input probability distributions, and he developed a four-hour videotape on simulation with the society for Manufacturing Engineers. Dr. Law wrote a regular column on simulation for Industrial Engineering magazine from 1990 through 1991.

He has been a tenured faculty member and has taught simulation at the University of Wisconsin and the University of Arizona. Dr. Law has a Ph.D. in Industrial Engineering and Operations Research from the University of California at Berkeley.

JOHN S. CARSON is a founder and president of Carson/Banks \& Associates, Inc., a firm specializing in 
simulation model development, services, and training. $\mathrm{He}$ has developed numerous large-scale simulation models with application to manufacturing, material handling, warehousing, distribution, transportation and rapid transit, port operations and shipping, medical delivery systems, and reservations systems. He has served on the faculties of the Georgia Institute of Technology and the University of Florida. With Jerry Banks, he is a co-author of the widely-used texts Discrete-Event System Simulation (Prentice-Hall, 1984) and Getting Started with GPSS/H (Wolverine Software Corp., 1989). He is a member of IIE, ORSA, and TIMS. Dr. Carson received his Ph.D. in Industrial Engineering and Operations Research from the University of Wisconsin at Madison (1978).

JERRY G. FOX is manager of Simulation and Integration for Boeing Commercial Airplane Group's Manufacturing Research and Development. He received his BS in general engineering from the U.S. Military Academy in 1966 and his MBA at the University of Tennessee in 1971. He has over 15 years experience implementing real-time process management and control systems in the U.S. and Japan. The central thrust of his group is advancement of simulator technologies so complex manufacturing applications can be evaluated faster with less emphasis on programming skills.

STEPHEN K. HALLADIN is a senior engineer at Boeing Commercial Airplane Group in Seattle, Washington. $\mathrm{He}$ has a BS degree in electrical engineering from Oregon State University. Joining Boeing in 1979, Mr. Halladin has worked as a member of the Manufacturing Research and Development organization staff. In his current position, he is responsible for transferring simulation technology to line organizations by providing consultation and project support.

KENNETH J. MUSSELMAN is Vice President of Services for Pritsker Corporation. For more than fifteen years, Dr. Musselman has been active in simulation consulting. He has conducted projects in a diversity of fields, including aerospace, automotive, communications, computers, consumer products, criminal justice, electronics, petroleum, and transportation. His specialty is in the design and scheduling of manufacturing systems. He served as General Chair for the 1989 Winter Simulation Conference.

ONUR M. ULGEN is a senior consultant at the Production Modeling Corporation, a Detroit based simulation services company. $\mathrm{He}$ is also a professor of
Industrial and Manufacturing Systems Engineering at the University of Michigan-Dearborn. He received his Ph.D. degree in Industrial Engineering from Texas Tech University in 1979. His present consulting and research interests include object-oriented simulation program generators, scheduling, and project management. Dr. Ulgen is a member of IIE, TIMS/ORSA, SCS, PMI, and APICS. 